\title{
Gaia DR1 completeness within 250 pc \& star formation history of the Solar neighbourhood
}

\author{
Edouard J. Bernard \\ Université Côte dAzur, OCA, CNRS, Lagrange, France \\ email: ebernard@oca.eu
}

\begin{abstract}
We took advantage of the Gaia DR1 to combine TGAS parallaxes with Tycho-2 and APASS photometry to calculate the star formation history (SFH) of the solar neighbourhood within $250 \mathrm{pc}$ using the colour-magnitude diagram fitting technique. We present the determination of the completeness within this volume, and compare the resulting SFH with that calculated from the Hipparcos catalogue within $80 \mathrm{pc}$ of the Sun. We also show how this technique will be applied out to $\sim 5 \mathrm{kpc}$ thanks to the next Gaia data releases, which will allow us to quantify the SFH of the thin disc, thick disc and halo in situ, rather than extrapolating based on the stars from these components that are today in the solar neighbourhood.
\end{abstract}

Keywords. Hertzsprung-Russell diagram, Galaxy: disk, Galaxy: evolution, Galaxy: formation, solar neighbourhood

\section{Introduction}

Disc galaxies dominate the stellar mass density in the Universe, yet the details of their formation and evolution are still poorly understood. Even in the Milky Way, for which we have access to a tremendous amount of information, the onset of star formation and the evolution of the star formation rate (SFR) of the Galactic components are all but unknown. This seriously hinders the interpretation of the available observations and the quantitative comparisons with galaxy formation models. However, the details of the formation of stellar systems are encoded in the distribution of the stars in deep colourmagnitude diagrams (CMDs). Their star formation history ( $\mathrm{SFH}$ ), that is, the evolution of both the SFR and the metallicity from the earliest epoch to the present time, can thus be recovered using the robust CMD-fitting technique. This technique requires the precise knowledge of the intrinsic luminosity of each star, and therefore its distance. In the coming years, Gaia will deliver distances and proper motions for over a billion stars out to $\sim 10 \mathrm{kpc}$, thus covering all the structural components of our Galaxy. For the first time, this opens the possibility to map the spatial and temporal variations of the SFH back to the earliest epochs. We illustrate this potential by exploiting the Gaia DR1 and TGAS parallaxes for over 2 million stars in the solar neighbourhood (Gaia Collaboration et al. 2016, Lindegren et al. 2016), and calculating the SFH of the Milky Way disc within $250 \mathrm{pc}$ of the Sun.

\section{The solar neighbourhood CMD: photometry and completeness}

While TGAS provides accurate parallaxes and G-band magnitudes for over 2 million stars, no colour information is available. On the other hand, the Tycho-2 catalogue does include $B_{T}, V_{T}$ for all TGAS stars, but the photometric quality quickly degrades at fainter magnitudes. We thus cross-matched TGAS with the Tycho-2, Hipparcos, and APASS (Henden et al. 2012) DR9 catalogues, after homogenizing their photometry to 

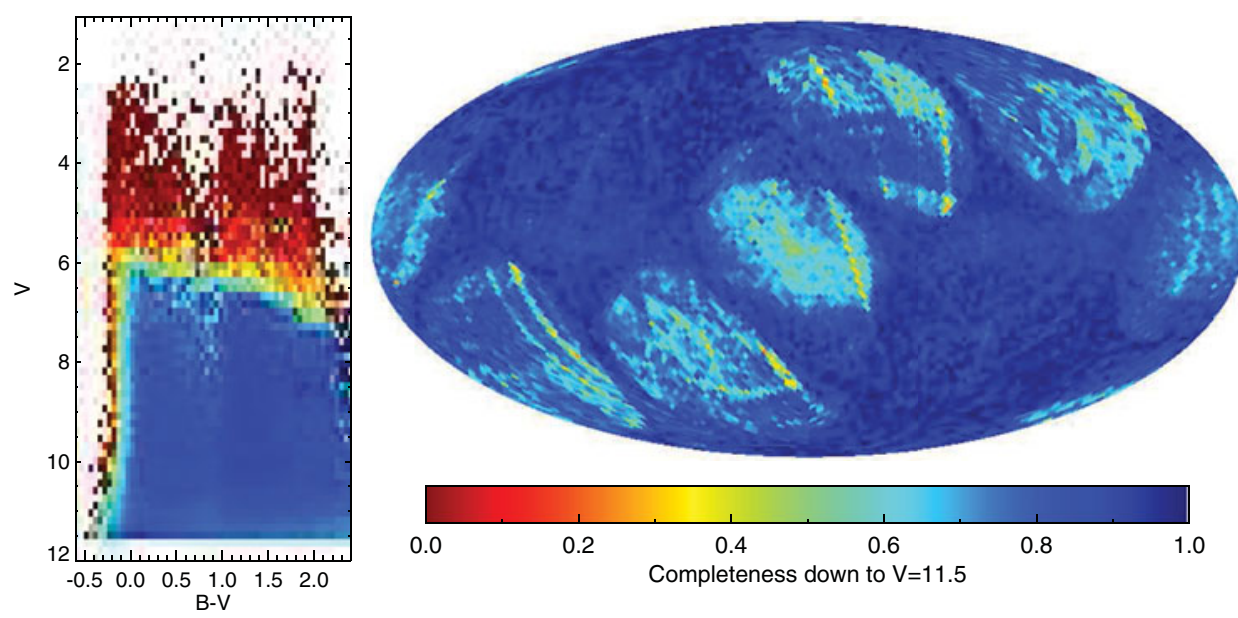

Figure 1. TGAS completeness down to $V=11.5$ relative to Tycho-2 in colour-magnitude space (left) and in galactic coordinates (right), before the completeness corrections.

the Johnson $B$ and $V$ filters. For stars appearing in more than one catalogue, a weighted mean magnitude was calculated for each filter.

A further step before the CMD-fitting can be applied is a robust quantification of the completeness as a function of colour and magnitude. According to $\mathrm{H} \varnothing \mathrm{g}$ et al. (2000), the Tycho-2 completeness is over $90 \%$ down to $V \sim 11.5$, but decreases quickly at fainter magnitudes, so we only kept stars brighter than this limit. Even though TGAS is based on Tycho-2, about $20 \%$ of the stars from the latter catalogue are missing in TGAS, which means that we have no robust parallaxes for these stars. This is illustrated in Figure 1, which presents the completeness of TGAS versus Tycho-2 as a function of both colourmagnitude (left) and spatial coordinates (right). The left panel shows that a significant fraction of the stars brighter than $V \sim 6.5$ or bluer than $B-V \sim 0$ are missing in TGAS; however, most of these stars have Hipparcos parallaxes, which we combined with those from TGAS to erase completeness variations as a function of colour and magnitude. The right panels shows that completeness is below $\sim 60 \%$ in about half of the sky due to the lower number of Gaia transits. Unfortunately, the completeness function in these regions is too complex to correct; we therefore simply excised $57 \%$ of the sky coverage where completeness down to $V \sim 11.5$ was $<90 \%$.

Finally, to obtain an accurate SFH back to the earliest epoch of star formation, a CMD reaching the oldest main-sequence turn-off (oMSTO, at $M_{V}=4.5$ ) is required. Given the completeness limits described above, the volume in which the SFH can be calculated is therefore limited to a distance modulus of $\left(V-M_{V}\right)=7$, corresponding to $250 \mathrm{pc}$. The resulting CMD, shown in the left panel of Figure 2, contains $~ 148,000$ stars and is mostly complete down to the oMSTO within $250 \mathrm{pc}$.

\section{SFH calculation and results}

The preliminary SFH has been calculated using the technique of synthetic CMDfitting following the methodology presented in Bernard et al. (2012, 2015a, 2015b). The synthetic CMD from which we extracted the simple stellar populations' CMDs is based on the BaSTI stellar evolution library (Pietrinferni et al. 2004). It contains $2 \times 10^{7}$ stars and was generated with a constant SFR over wide ranges of age and metallicity: 0 to 15 Gyr old and $0.0001 \leqslant \mathrm{Z} \leqslant 0.03$ (i.e. $-2.3 \leqslant[\mathrm{Fe} / \mathrm{H}] \leqslant 0.26$, assuming $\mathrm{Z}_{\odot}=0.0198$; 

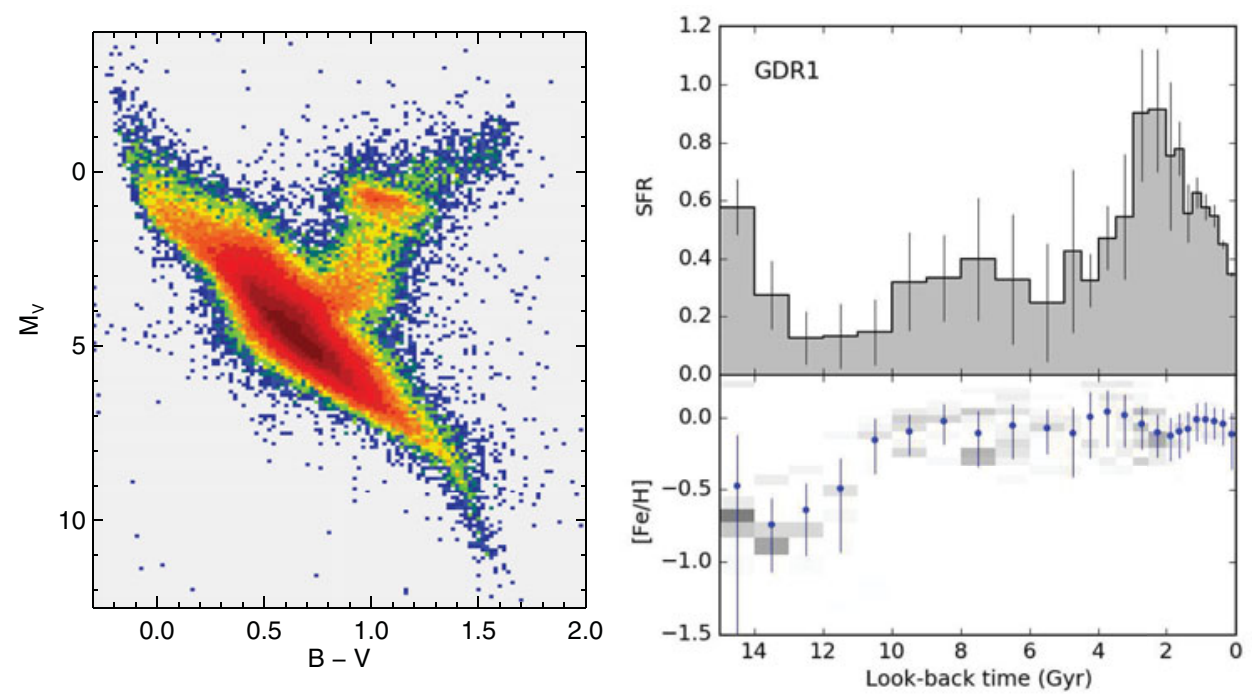

Figure 2. Left: CMD for the solar neighbourhood within $250 \mathrm{pc}$, which is $\sim 94 \%$ complete down to $\mathrm{M}_{V}=4.5$. Right: Resulting SFH, showing the evolution of the SFR (top) and metallicity (bottom) as a function of time.

Grevesse \& Noels 1993). We adopted a Kroupa (2002) initial mass function, and assumed a fraction of unresolved binary systems in TGAS of $10 \%$ with mass ratios between 0 and 1. Further tests with different fractions of binaries, a wider range of metallicity, and different prescriptions for the simulated photometric and parallactic uncertainties are necessary to better understand the possible systematic uncertainties.

While the full photometric uncertainties due to various observational effects are typically estimated using artificial stars tests on the original images (e.g. Gallart et al. 1999), this approach would be intractable for large photometric surveys, not to mention impossible in the case of Gaia for which (most of) the images are not sent back to Earth. Instead, we relied on the distributions of photometric errors as a function of colour and magnitude provided in the Tycho-2, Hipparcos, and APASS catalogues to simulate the uncertainties in the synthetic CMD.

The resulting SFH is presented in the right panel of Figure 2: the top and bottom plots show the evolution of the SFR and metallicity, respectively, as a function of time. Note that since the SFH was reconstructed based on the stars that are located today within the solar neighbourhood, the possible effects of secular evolution in discs (e.g. radial migrations, disc heating; e.g. Sellwood \& Binney 2002, van der Kruit \& Freeman 2011) have to be taken into account when interpreting the plots shown in Figure 2.

The SFH shows a roughly constant SFR for the first 10 Gyr or so, with a slight enhancement in the past 4 Gyr. This is in excellent agreement with the SFH calculated from Hipparcos data within a smaller volume ( $\sim 80 \mathrm{pc}$; Vergely et al. 2002 , Cignoni et al. 2006), and with the known excess of stars younger than $\sim 4$ Gyr old in the solar neighbourhood (e.g. Edvardsson et al. 1993, Anders et al. 2017). The age-metallicity relation (AMR), shown in the bottom-right panel of Figure 2, is mostly flat for the past 10 Gyr. Only the oldest stars show a lower mean metallicity $([\mathrm{Fe} / \mathrm{H}] \sim-0.7)$, which may correspond to the thick disc population. This is fully consistent with the independent results from other groups using different methods (e.g. Casagrande et al. 2011, Haywood et al. 2013, Bergemann et al. 2014). 


\section{Conclusions and future prospects}

Taking advantage of the Gaia DR1 providing parallaxes for over 2 million stars in the solar neighbourhood, we produced a deep CMD that is mostly complete down to the magnitude of the oMSTO within $250 \mathrm{pc}$ from the Sun. We applied the CMD-fitting technique to reconstruct the SFH of the Milky Way local thin disc. The preliminary results are fully consistent with those obtained previously using the Hipparcos data, despite the difficulty of dealing with the complex completeness function and photometric uncertainties from different catalogues. This is very promising for the prospects of using the same technique to upcoming Gaia data releases. With parallaxes and homogeneous photometry in 3 bands $(G, B P, R P)$ for $>10^{9}$ stars, and not limited by the poorly understood completeness function of an input catalogue like Tycho-2 was, it will allow us to extend this analysis out to about $5 \mathrm{kpc}$, and therefore to quantify the SFH of the thin disc, thick disc and halo in situ - rather than extrapolating based on the stars from these components that are today in the solar neighbourhood - and its spatial variations.

\section{Acknowledgements}

EJB acknowledges support from the CNES postdoctoral fellowship program. This work has made use of data from the European Space Agency (ESA) mission Gaia (https://www.cosmos.esa.int/gaia), processed by the Gaia Data Processing and Analysis Consortium (DPAC, https://www.cosmos.esa.int/web/gaia/dpac/consortium). Funding for the DPAC has been provided by national institutions, in particular the institutions participating in the Gaia Multilateral Agreement.

\section{References}

Anders, F., Chiappini, C., Rodrigues, T. S., et al. 2017, A\&A, 597, A30

Bergemann, M., Ruchti, G. R., Serenelli, A., et al. 2014, A\&A, 565, A89

Bernard, E. J., Ferguson, A. M. N., Barker, M. K., et al. 2012, MNRAS, 420, 2625

Bernard, E. J., Ferguson, A. M. N., Chapman, S. C., et al. 2015b, MNRAS, 453, L113

Bernard, E. J., Ferguson, A. M. N., Richardson, J. C., et al. 2015a, MNRAS, 446, 2789

Casagrande, L., Schönrich, R., Asplund, M., et al. 2011, AESA, 530, A138

Cignoni, M., Degl'Innocenti, S., Prada Moroni, P. G., \& Shore, S. N. 2006, A\& A, 459, 783

Edvardsson, B., Andersen, J., Gustafsson, B., et al. 1993, A\&A, 275, 101

Gaia Collaboration, Brown, A. G. A., Vallenari, A., et al. 2016, A\&SA, 595, A2

Gallart, C., Freedman, W. L., Aparicio, A., Bertelli, G., \& Chiosi, C. 1999, AJ, 118, 2245

Grevesse N., Noels A., 1993, in Prantzos N., Vangioni-Flam E., Cassé M., eds, Origin and Evolution of the Elements. Cambridge Univ. Press, Cambridge, p. 15

Haywood, M., Di Matteo, P., Lehnert, M. D., Katz, D., \& Gómez, A. 2013, A $\& A$, 560, A109

Henden, A. A., Levine, S. E., Terrell, D., Smith, T. C., \& Welch, D. 2012, Journal of the American Association of Variable Star Observers (JAAVSO), 40, 430

Høg, E., Fabricius, C., Makarov, V. V., et al. 2000, A\&\&A, 355, L27

Kroupa P., 2002, Sci, 295, 82

Lindegren, L., Lammers, U., Bastian, U., et al. 2016, A\&A, 595, A4

Pietrinferni A., Cassisi S., Salaris M., Castelli F., 2004, ApJ, 612, 168

Sellwood, J. A. \& Binney, J. J. 2002, MNRAS, 336, 785

van der Kruit, P. C. \& Freeman, K. C. 2011, ARA\&A, 49, 301

Vergely, J.-L., Köppen, J., Egret, D., \& Bienaymé, O. 2002, A\& $A$, 390, 917 